across the lifespan, is now underway ${ }^{6}$. These cutting-edge, high-resolution data may suggest temporal and regional targets for future RNA sequencing and pathway analyses aimed at identifying cell-specific and region-specific gene expression changes relevant to neuroregeneration in humans and their associated mouse models.

The current study by Sas et al. reveals neuroregeneration that is mediated by the immature neutrophil subset in the optic nerve and spinal cord. Whether other regions in the CNS are similarly affected remains unknown. Even in a healthy brain, there are gene expression differences from one brain region to another in astrocytes ${ }^{7,8}$, microglia $^{9}$ and oligodendrocytes ${ }^{10}$. A difference in changes in gene expression in these cells from one brain region to another during injury has also been demonstrated $^{5,9,11}$. For example, astrocytes in the spinal cord and optic nerve varied to some degree from each other but varied markedly as compared to the cerebral cortex, hippocampus and cerebellum ${ }^{11,12}$. Since astrocytes and microglia are involved in the recruitment of immune cells from the blood into the CNS, this finding may explain why the infiltration of neutrophils has been shown to differ from one brain region to another in neuroinflammatory models ${ }^{13}$. Whether findings in the current paper about the optic nerve and spinal cord are applicable to other CNS regions within the brain warrants further investigation. This discovery could help to determine which human diseases are amenable to clinical translation of neuroregeneration induced by neutrophils of the immature subtype.

There are several implications for the translation of the findings in the current paper to humans. Human conditions warranting potential study for translation would include optic nerve and spinal cord traumatic injuries, glaucoma, optic neuritis in multiple sclerosis and neuromyelitis optica. While demonstrating the preservation of neurons and axonal regeneration is a widely used outcome to show a treatment is beneficial in mouse models of optic nerve injury, clinical outcomes showing preservation of vision are also needed to complement optic nerve and retinal ganglion cell findings. Improvement in walking ability is also needed to accompany improvement in spinal cord neuropathology. It is critical to discern whether the results of immature neutrophil treatment are robust enough to induce clinical efficacy in preclinical mouse models before proceeding to clinical studies in humans. Also, adverse effects related to physical injuries or untoward local immune reactions during intraocular injections of immature neutrophils may preclude translation to humans. This concern would shift the clinical relevance of these results from the adoptive transfer of cells to the injection of the combination of growth factors from these cells. However, growth factors were only partially protective, and whether cell-to-cell contact played a role in neuroregeneration remained unclear. Finally, a practical clinical implication of the current study is the use of immunosuppressive treatments with corticosteroids for traumatic or neuroinflammatory optic nerve and spinal cord injury. Perhaps one should weigh the possibility of gaining short-term benefit from reducing edema and inflammation against the long-term consequences of neuronal sparing and axonal regeneration. Whether corticosteroid treatment after injury affects deleterious, mature ' $\mathrm{N1}$ ' neutrophils and protective, immature 'N2' neutrophils equally or differentially is a question worth pursuing in future studies.

\section{Rhonda Voskuhl(iD)}

Department of Neurology, David Geffen School of Medicine, University of California, Los Angeles, Los Angeles, CA, USA.

$\bigotimes_{\mathcal{e} \text {-mail: rvoskuhl@mednet.ucla.edu }}$

Published online: 2 November 2020

https://doi.org/10.1038/s41590-020-00821-0

References

1. Sas, A. R. et al. Nat. Immunol. https://doi.org/10.1038/s41590020-00813-0 (2020).

2. Miron, V. E. et al. Nat. Neurosci. 16, 1211-1218 (2013).

3. Ruckh, J. M. et al. Cell Stem Cell 10, 96-103 (2012).

4. Moyon, S. et al. J. Neurosci. 35, 4-20 (2015).

5. Voskuhl, R. R. et al. Proc. Natl Acad. Sci. USA 116, 10130-10139 (2019).

6. Bookheimer, S. Y. et al. Neuroimage 185, 335-348 (2019).

7. Chai, H. et al. Neuron 95, 531-549.e9 (2017).

8. Khakh, B. S. \& Sofroniew, M. V. Nat. Neurosci. 18, 942-952 (2015)

9. Grabert, K. et al. Nat. Neurosci. 19, 504-516 (2016).

10. Marques, S. et al. Science 352, 1326-1329 (2016).

11. Itoh, N. et al. Proc. Natl Acad. Sci. USA 115, E302-E309 (2018).

12. Tassoni, A. et al. Sci. Rep. 9, 10010 (2019).

13. Yan, Z. et al. JCI Insight 5, e126520 (2019).

Competing interests

The author declares no competing interests.

\title{
Overlapping B cell pathways in severe COVID-19 and lupus
}

\author{
An exaggerated extrafollicular B cell response characteristic of active systemic lupus erythematosus also \\ characterizes the B cell response to SARS-CoV-2 in those with severe COVID-19.
}

\section{A. Darise Farris and Joel M. Guthridge}

\section{$\mathrm{T}$} o understand the immunologic underpinnings of severe COVID-19, Woodruff and colleagues from the Sanz group ${ }^{1}$ used deep immunophenotyping to compare B cell responses in patients hospitalized in the intensive care unit (ICU) to those of mildly affected outpatients. Despite poor outcomes, the severely affected patients paradoxically produced strong antibody-secreting cell (ASC) responses and neutralizing antiviral antibodies. However, immunophenotyping revealed that the source of the ASC response differed between severe and mild cases. In an interesting parallel between severe COVID19 and autoimmune disease, the severely affected individuals showed exaggerated extrafollicular (EF) B cell responses 


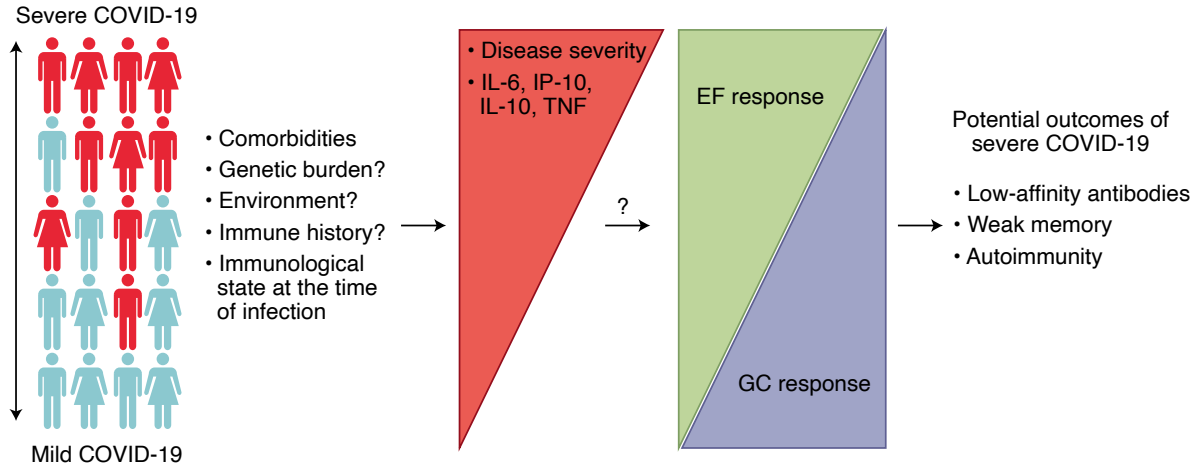

Fig. 1 | Does intrinsic immune bias predispose to severe COVID-19? In a largely AA cohort, Woodruff et al. show that subjects with severe COVID-19 make exaggerated extrafollicular (EF) B cell responses, which are also observed in AAs with active SLE. This raises the question of whether an increased propensity for inflammation contributes to the risk for severe COVID-19. Among the elevated soluble mediators in severe COVID-19 are IL-6, IP-10, IL-10 and TNF, which may promote EF and suppress $\mathrm{GC}$ responses. Other studies showing reduced or absent $\mathrm{GC}$ responses and EF-response-associated autoantibodies in severe SARS-CoV-2 infection support an EF bias in the adaptive immune response in people with a severe disease course. Potential deleterious outcomes of an EF-biased response range from inadequate immunologic memory to possible autoimmune sequelae. Understanding the precise cellular mechanisms leading to innate bias in the adaptive immune response to severe COVID-19 could lead to therapeutic approaches that restore the balance between EF and GC responses.

that were strikingly similar to those the group had previously observed in African Americans (AAs) with active systemic lupus erythematosus (SLE) ${ }^{2}$.

EF responses, which are prominent in early viral and bacterial infections in animal models, occur outside of germinal centers (GCs). These responses are traditionally thought to produce antibodies with fewer somatic mutations and may elicit less robust immunologic memory as compared to GC responses, which typically produce high-affinity, highly somatically mutated antibodies and strong memory ${ }^{3}$. In humans with severe COVID-19 or severe SLE, the ASC response appears to originate primarily from $\mathrm{CD} 11 \mathrm{c}^{+}$'activated naive' cells, and at least one subset of these gives rise to ASCs through an intermediate 'double-negative (DN)2' population that lacks expression of IgD (naive marker), CD27 (memory marker), CXCR5 and CD21. These populations were significantly and coordinately expanded in both the hospitalized patients with COVID-19 ${ }^{1}$ and those with active SLE ${ }^{2}$. In the COVID-19 group, these populations were associated with elevated plasma concentrations of the inflammatory biomarker C-reactive protein (CRP), the chemokine IP-10 (also known as CXCL10), and the cytokine interleukin (IL)-6. Moreover, many clonally related B cell receptors sequenced from the ICU patients showed few somatic mutations, characteristic of EF responses. Highly imbalanced EF:GC responses in severe SARS-CoV-2 infection were also recently corroborated by the Pillai group, who showed that GCs, GC B cells and $\mathrm{T}$ follicular helper cells were absent or severely reduced in post-mortem secondary lymphoid tissues of those who died from COVID $-19^{4}$. This group saw similar findings in blood, and the results were correlated with high concentrations of CRP. They also detected tumor necrosis factor (TNF) in the secondary lymphoid organs of those who succumbed to COVID-19 but not in tonsil tissue from SARS-CoV-2-uninfected individuals. Notably, TNF can suppress GC responses in animal models.

A key unanswered question is whether the exaggerated EF response detected in severe COVID-19 plays an active role in disease pathogenesis or whether it merely serves as a biomarker of the strong inflammatory responses that occur in those who are severely affected. Direct pathogenic effects of this response could include EF B cell cytokine production, antigen presentation or autoantibody production. Alternatively, the skewed EF response may be a consequence of elevated inflammatory mediators. Consistent with this, several inflammatory proteins elevated in severe COVID-19 have previously been associated with EF responses. Among these are IL-6, IL-10 and IP-10 ${ }^{5}$. Woodruff and colleagues cite multiple papers linking elevated concentrations of IL- 6 and IP-10 with EF responses, and we showed that IL- 6 deficiency could prevent abnormal Toll-like receptor 7 (TLR7)-driven EF responses, anti-La autoantibodies and disease in a mouse model of lupus ${ }^{6}$. Mechanistic studies measuring EF B cell populations in COVID19 clinical trials targeting IL-6 signaling could reveal whether IL- 6 is a key cytokine causing the skewed EF response. Elevated IL-10 could also be involved in propelling the EF:GC imbalance by inhibiting the function of $\mathrm{T}$ follicular helper cells that drive GC responses ${ }^{7}$. Elevated IL-10 in SARS-CoV-2 infection could arise from both hyper-responsive myeloid cells and newly described subsets of IL-10-producing T helper cells that are elevated in SLE and promote EF responses (reviewed in ref. ${ }^{7}$ ). The role of IL-10 in SARS-CoV-2 infection may not be simple, however, as Woodruff et al. also reported that those with mild disease had an expanded transitional B cell population. This population is known to contain IL-10-producing regulatory B cells. Therefore, IL-10 may play deleterious or protective roles, depending on which cellular populations express it. Further studies will be needed to determine the extent to which skewed EF responses promote or reflect severe COVID-19.

In the Woodruff study, the vast majority (83\%) of patients with COVID-19 who were severely affected were AAs, while none of the mildly affected individuals were AAs. Given the similar EF-dominant responses between AAs with severe COVID-19 and AAs with active, possibly more severe SLE, one may speculate that AA individuals may be disproportionately affected by COVID19 due in part to an elevated immunologic propensity for increased inflammation and EF responses. However, whether the exaggerated $\mathrm{EF}$ responses in those with severe COVID-19 are specific to AAs will need to be verified in larger groups of subjects that include AAs with mild disease and non-AAs with severe COVID-19, as well as mildly and severely affected Hispanic and Native American populations, groups that also had elevated hospitalization rates ${ }^{8}$. Other populations that could be examined include children with severe disease and those with multisystem inflammatory syndrome.

While many studies have focused primarily on comorbidities and socioeconomic factors in explaining the disproportionate disease severity in AAs, recent large studies revealed that AAs have higher rates of hospitalization, even after correcting for these factors ${ }^{9}$. SLE tends to be more severe in AAs as compared to European Americans, and the genetic risk for SLE varies by ethnicity. Therefore, it is conceivable that an increased burden of genetic risk for inflammatory and EF 
responses could be a contributing factor to severe COVID-19 and severe SLE in subsets of AA individuals. Signals from TLR7, TLR9 and soluble cytokine factors like BAFF, APRIL, IL-21 and IL-10 promote EF B cell responses ${ }^{3}$. Coincidentally, polymorphisms in multiple genes in these pathways (for example, TLR7 itself, IRF5 and several genes affecting the transcription factor NF- $\mathrm{B}$ ), as well as others with suspected roles in promoting $\mathrm{EF}$ responses on the basis of animal models (such as $B L K$, $B A C H 2, E T S 1$ and $L Y N$ ) are associated with SLE risk. There is emerging evidence that an increased burden of genetic risk correlates with renal disease severity in $\mathrm{SLE}^{10}$. Thus, a disproportionate increase in the genetic predisposition toward elevated inflammation and EF responses in AAs with severe SLE is plausible but has not yet been shown. Further studies will be needed to dissect the extent to which genetic burden, immunologic history, immunologic state at the time of infection, comorbidities and other environmental influences contribute to an elevated risk for severe COVID-19 in AA individuals. Assessing those with severe versus mild COVID-19 for SLE risk alleles would help to address whether the high inflammatory and EF responses in these two conditions share roots in genetic risk.

Potential molecular players mediating exaggerated $\mathrm{EF}$ responses in severe COVID-19 as well as possible downstream consequences are outlined in Fig. 1. If the skewed EF response results in lower affinity antibodies that are less effective at viral neutralization or clearance, then an intrinsic bias toward such a response could be a driving factor contributing to disease severity. However, many studies are showing that those with severe COVID19 have longer lasting anti-SARS-CoV-2 antibodies with a higher titer than those with mild disease. This finding could be directly related to viral titers and the amount of antigen to which the immune system is exposed. The conventional understanding of EF and GC responses suggests that another potential consequence is a lack of durable immunologic memory. Only time will tell exactly how durable memory B cells responses are following recovery from severe COVID-19. This possibility needs to be carefully monitored in future studies and will, we hope, be addressed with vaccination, whereby responses are not necessarily skewed by hyperinflammatory conditions. A third possible deleterious consequence is rheumatic disease sequelae. Although EF responses in AA patients with lupus were associated with anti-Sm and anti-RNP autoantibodies ${ }^{2}$, recent reports document a high prevalence and high levels of anti-Ro and anti-La lupus-like autoantibodies in Asian patients with severe COVID-19 $9^{11,12}$. There is evidence that these autoantibodies, which also occur in SLE and in related diseases like Sjögren's syndrome, originate from the EF pathway. Whether some individuals with severe COVID-19 who are also genetically predisposed to rheumatic diseases like SLE and Sjögren's will go on to develop these autoimmune conditions is an open question. Viral triggering of these diseases has long been suspected but is difficult to prove.

In summary, Woodruff et al. highlight a B cell pathway important in both severe COVID-19 and active SLE. This observation may accelerate the pace of understanding and treating both conditions.

\section{A. Darise Farris (D) $\times$ and Joel M. Guthridge (1D)}

Arthritis and Clinical Immunology Program, Oklahoma Medical Research Foundation, Oklahoma City, OK, USA.

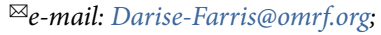
Joel-Guthridge@omrf.org

Published online: 2 November 2020 https://doi.org/10.1038/s41590-020-00822-z

References

1. Woodruff, M. C. et al. Nat. Immunol. https://doi.org/10.1038/ s41590-020-00814-z (2020).

2. Jenks, S. A. et al. Immunity 49, 725-739.e6 (2018).

3. Jenks, S. A., Cashman, K. S., Woodruff, M. C., Lee, F. E. \& Sanz, I. Immunol. Rev. 288, 136-148 (2019).

4. Kaneko, N. et al. Cell https://doi.org/10.1016/j.cell.2020.08.025 (2020).

5. Laing, A. G. et al. Nat. Med. https://doi.org/10.1038/s41591-0201038-6 (2020).

6. Maier-Moore, J. S. et al. Arthritis Rheumatol. 66, 2521-2531 (2014).

7. Geginat, J. et al. Semin. Immunol. 44, 101330 (2019).

8. Kim, L. et al. MMWR Morb. Mortal. Wkly Rep. 69, 1081-1088 (2020)

9. Price-Haywood, E. G., Burton, J., Fort, D. \& Seoane, L. N. Engl. J. Med. 382, 2534-2543 (2020).

10. Chen, L. et al. Hum. Mol. Genet. 29, 1745-1756 (2020).

11. Fujii, H. et al. Clin. Rheumatol. https://doi.org/10.1007/s10067020-05359-y (2020).

12. Zhou, Y. et al. Clin. Transl. Sci. https://doi.org/10.1111/cts.12805 (2020).

Competing interests

The authors declare no competing interests.

\title{
CRELD1 controls T cell homeostasis
}

\author{
The natural variability of CRELD1 expression identified in comprehensive data sets from healthy individuals helps \\ predict and establish its role in regulating $T$ cell homeostasis.
}

\section{Fotini Gounari}

$\mathrm{C}$ ysteine-rich with EGF-like domains 1 (CRELD1) is a ubiquitously expressed transmembrane protein that is critically required for embryonic heart development, but its roles in other tissues and in adult life remain elusive. In this issue of Nature Immunology, Bonaguro et al. ${ }^{1}$ demonstrate how analyzing the expression variance of CRELD1 in healthy individuals guided their approach to understanding its physiological functions in T cells. The authors considered extremes of CRELD1 expression in available datasets from heathy humans as quasi loss- and gain-of-function conditions. Their studies showed that CRELD1 modulates NFAT and
Wnt signaling in T cells and is required for maintaining T cell homeostasis (Fig. 1).

The new approach taken by the authors was inspired by the availability of a number of comprehensive datasets from cohorts of healthy humans that correlate variations in the transcriptome, phenotype and cellular functions of immune cells with 